\section{Análise da demanda de casos de hanseníase aos serviços de saúde através do uso de técnicas de análise espacial}

\author{
Spatial analysis of leprosy cases treated at public \\ health care facilities in Brazil
}

\author{
${ }^{1}$ Centro de Pesquisas Aggeu \\ Magalhães, Fundação \\ Oswaldo Cruz, Recife, Brasil. \\ 2 Escola Nacional de Saúde \\ Pública Sergio Arouca, \\ Fundação Oswaldo Cruz, \\ Rio de Janeiro, Brasil. \\ Correspondência \\ T. M. Lapa \\ Centro de Pesquisas Aggeu \\ Magalhães, Fundação \\ Oswaldo Cruz. \\ Av. Moraes Rego s/n, Cidade \\ Universitária, Recife, $P E$ \\ 50670-420, Brasil. \\ tiago@cpqam.fiocruz.br
}

\begin{abstract}
Hansen disease or leprosy is a major endemic disease in Brazil. Well-designed strategies, including decentralization of basic care, are needed to reduce its prevalence. The article begins by describing the structure and supply of services for treating leprosy cases in the country, after which it analyzes the trends in epidemiological and operational indicators, comparing the periods before and after decentralization of services to the municipal (local) level. Finally, spatial analysis allowed identifying the territorial distribution of this endemic and analyzing the pattern of geographic areas according to care provided by health facilities and its evolution. Based on the location of the geographic centers in the census tracts by place of residence, and using spatial smoothing technique based on Kernel estimation, the study constructed domain areas of care for each health facility or unit. Following municipalization of care, there was an increase in the detection and treatment by the municipalities themselves, reducing patient evasion to neighboring counties and causing changes in demand trends, with an increase in use of services by the clientele and important alterations in the epidemiological and operational indicators.
\end{abstract}

Leprosy; Spatial Analysis; Health Services Accessibility
Tiago Maria Lapa 1

Maria de Fátima P. Militão de Albuquerque 1

Marilia Sá Carvalho 2

José Constantino Silveira Júnior 1

\section{Introdução}

Tanto pela sua magnitude quanto pelas seqüelas que a doença acarreta e conseqüentes transtornos emocionais e sociais para o doente e sua família, a hanseníase é, ainda hoje, um dos mais sérios problemas de saúde pública do Brasil. O projeto brasileiro de eliminação da hanseníase, do ponto de vista da infra-estrutura dos serviços, tem se fundamentado basicamente em uma proposta de ampliação da rede de diagnóstico e atenção ao paciente, mediante a descentralização das atividades para os serviços de atenção básica à saúde. Paralelo a isto, a divulgação dos sinais e sintomas da doença para a população em geral constitui-se um instrumento para a eliminação da endemia 1,2 .

As mudanças propostas nesse novo modelo de organização dos serviços de saúde pretendem romper com a tendência do atendimento espontâneo - aquele voltado às pessoas que, na dependência de seu grau de percepção ou sofrimento, procuram os serviços de saúde - para proporcionar uma oferta organizada em função dos principais agravos e grupos populacionais prioritários. No que diz respeito às intervenções sobre a hanseníase, para o planejamento dos recursos a serem alocados e para o diagnóstico das necessidades da população, a definição de área de abrangência de uma unidade de saúde, e o conseqüente conhecimento da população adscrita, é indispensável 3. 
Neste trabalho pretendemos avançar na discussão sobre área de abrangência, evitando a lógica administrativa de uma área previamente arbitrada e delimitando áreas com base na utilização efetiva pela população. Em síntese, a idéia é organizar e estimular a demanda a ser atendida a partir das necessidades de grupos populacionais residentes em um dado território, utilizando-se como instrumento para esse planejamento a sua real utilização. Neste contexto, contribuir para a avaliação da atuação do Programa Saúde da Família (PSF), que vem se transformando no eixo do processo de reorganização dos serviços.

Partindo-se do pressuposto de que a distribuição geográfica da ocorrência da hanseníase não é uniforme, em conseqüência dos diversos fatores que a influenciam 4,5,6,7, o planejamento da oferta de serviços de saúde é indispensável na perspectiva da eqüidade. Este planejamento deve considerar as necessidades de cada subgrupo da população visando a reduzir as desigualdades no estado de saúde entre grupos populacionais de distintas condições sociais 8,9.

Entende-se que a utilização dos serviços de saúde é equânime quando a maior parte de sua variação é explicada pela estrutura demográfica e de necessidade; e, o oposto, quando a maior parte daquela variação é explicada por aspectos da estrutura social 10. Pinheiro 11, em seu estudo sobre variações no uso dos serviços, encontrou indicativos de que pacientes de piores condições sociais têm menor chance de receber o tratamento recomendado. Barreiras também são impostas pela localização da residência. "Os serviços de saúde estão inevitavelmente concentrados em determinados lugares, conseqüentemente, são mais acessíveis às pessoas que estão mais próximas" 12 (p. 97).

O uso dos serviços de saúde é condicionado também pelo acesso aos mesmos 13. Neste trabalho, com o objetivo de estudar a questão do acesso, foi utilizada metodologia de identificação de "áreas de domínio" da demanda atendida, ou seja, territórios geográficos de origem da população que utiliza cada unidade de saúde incluída no estudo ${ }^{14}$. Diante do grande desafio que é a eliminação da hanseníase no Brasil, e em especial em algumas cidades do Nordeste brasileiro, este estudo tem o objetivo de identificar, mediante técnicas de análise espacial, os padrões da ocorrência da hanseníase e os padrões de utilização das unidades de saúde. Parte-se do pressuposto que os padrões de utilização dos serviços de saúde são ainda expressão da demanda espontânea, embora já possam estar a refletir os efeitos da municipalização enquanto tentativa de organizar essa demanda, em consonância com a análise da necessidade da população, neste caso, a ocorrência da hanseníase. Este conhecimento poderá vir a resultar na racionalização de recursos e adequação de serviços, com o conseqüente maior impacto sobre a redução da transmissão da hanseníase na busca de sua eliminação.

Sendo assim, neste trabalho foi analisada a distribuição espacial da demanda de casos de hanseníase por meio da utilização de Sistema de Informações Geográficas (SIG) e técnicas de análise espacial, para delimitar áreas de domínio das unidades de saúde e para visualizar/explorar o impacto da estratégia da descentralização do atendimento no Município de Olinda, comparando-se os períodos pré e pós-municipalização.

\section{Materiais e métodos}

\section{População de estudo}

Casos de hanseníase em residentes em Olinda, Pernambuco, Brasil, no período de 1991 a 2000, registrados no Sistema de Informações de Agravos de Notificação (SINAN), por cada unidade de saúde segundo localização pontual (residência) aproximada (centróide do setor censitário).

\section{Mapas}

Com base na malha censitária digital original, obtida com o Instituto Brasileiro de Geografia e Estatística (IBGE), utilizou-se o ArcView (ESRI, Redlands, Estados Unidos), incluindo como camadas de informações, além dos contornos dos polígonos dos setores censitários, seus centróides, a localização das unidades de saúde e postos de atendimento do PSF, incorporando ainda as divisões político-administrativas (bairros, áreas programáticas - AP - e distritos sanitários - DS) baseando-se nos descritores e croquis do IBGE e informações da Secretaria de Saúde e Planejamento de Olinda (Figura 1).

O envolvimento das 14 equipes do PSF no DS 1 e dez no DS 2, integradas às Unidades de Referência, implementadas ao longo do segundo qüinqüênio do período em estudo, envolvendo um grande contingente de profissionais de saúde com o controle da endemia, foram identificadas por representar um razoável esforço na busca da organização dos serviços de saúde do município.

\section{Análise espacial}

A unidade básica de estudo é a localização pontual dos casos de hanseníase, representada pelos centróides dos setores censitários aos quais foram georreferenciados, identificados pelas suas coordenadas. Estimou-se, para toda a região do 


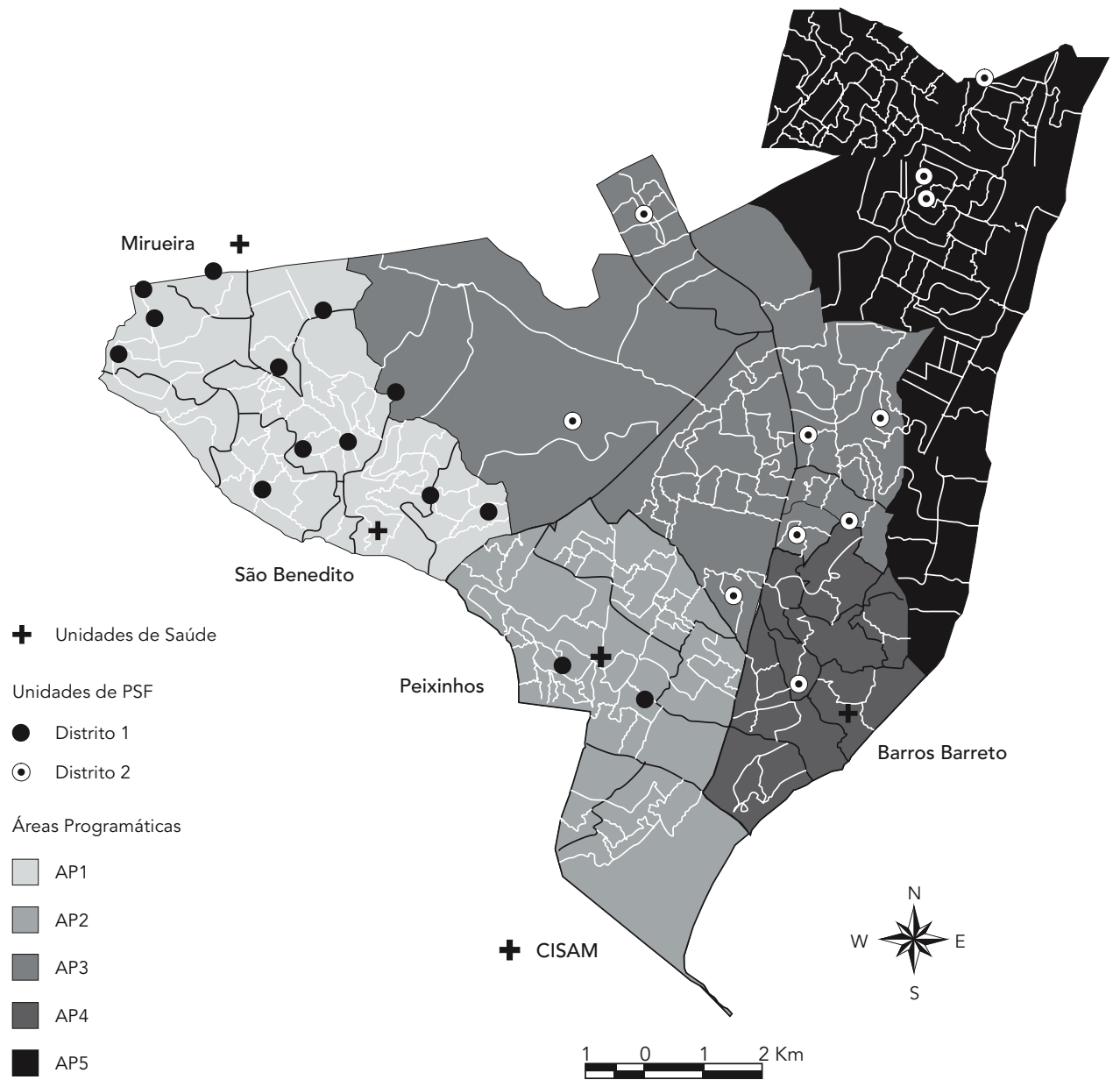

Além de Barros Barreto (referência para o distrito 2), São Benedito (referência para o distrito 1) e Peixinhos, as Unidades de Mirueira, em Paulista, e Centro Integrado de Saúde Amauri de Medeiros (CISAM), em Recife, estão localizadas no mapa por terem prestado significativos atendimentos aos residentes em Olinda.

estudo, a intensidade de ocorrência da doença, utilizando-se uma função de Kernel bi-dimensional 15, com correção do efeito de borda. Foi utilizada uma largura de banda de $1.000 \mathrm{~m}$ e grade regular de 200 X 200 células. Cada célula, de $50 \mathrm{~m} \times 50 \mathrm{~m}$, corresponde a um lugar $s$, que recebe valor estimado pela função, que pondera os pontos pela distância que estão de $s$, transformando o mapa de pontos em área contínua, que representa por meio de um gradiente suave as intensidades de ocorrência da hanseníase.

Construiu-se então mapa do "acesso" aos serviços de saúde através da matriz de máximos en- tre as proporções calculadas pelo quociente da intensidade de ocorrência de casos atendidos por cada unidade de saúde e a intensidade produzida pelo Kernel para o total de casos do município 11,14. Esta matriz de máximos permite mapear áreas de domínio de atendimento e de divisão do atendimento, onde nenhuma unidade de saúde atinge a proporção $50 \%$ de atendimento. Nestas, uma determinada unidade de saúde pode ter importância diferenciada e pode-se analisar temporalmente a evolução da cobertura das unidades.

Assim, foram construídos mapas qüinqüenais, identificando a unidade de saúde que 
obteve as máximas proporções de atendidos por áreas, permitindo identificar a evolução como prestadoras nestas áreas.

\section{Resultados}

Pode-se observar que, entre 1991 e 1994, o coeficiente de detecção estava estacionário em torno de 7,2/10 mil habitantes, aumenta para média de aproximadamente 11 entre 1996 e 2000, com valor intermediário em 1995 (Tabela 1).

Na Figura 2 apresenta-se o mapa com a estimativa de intensidade para a ocorrência da hanseníase, utilizando-se os 2.734 casos ocorridos em todo o período e georreferenciados.

A sobreposição das camadas com as delimitações das áreas programáticas e localização das unidades de saúde, permite uma visão preliminar identificando onde estão os maiores focos de ocorrência .

Os números da Tabela 2 mostram o expressivo crescimento dos atendimentos pelas principais unidades de Olinda, que saltam dos 591 no primeiro qüinqüênio para 1.111 no segundo. Podemos destacar ainda os 1.075 atendimentos por encaminhamento no segundo qüinqüênio, tipo de demanda que passou a ser predominante neste período, inclusive nas unidades de Olinda com o expressivo número de 548 atendimentos.

Reproduzindo o procedimento para os universos de atendimentos - a clientela - de cada uma das principais unidades de saúde, foi possí- vel classificar as áreas de acordo com as proporções de atendimentos pelas principais unidades, que construídas para cada um dos qüinqüênios possibilitou melhor observar a evolução do processo de atendimento dos casos.

Na Figura 3 estão representadas as evoluções qüinqüenais das áreas em que as principais unidades de atendimento exercem o domínio (atendem $50 \%$ ou mais dos casos) e onde exercem o maior atendimento a seus residentes.

Como pode ser observado, destaca-se a redução de áreas de forte predomínio, constatandose ainda uma grande divisão de atendimentos pelas unidades de saúde.

No DS 2, chama a atenção que a área de domínio dos atendimentos aos casos de hanseníase em seus residentes, exercido por sua unidade de referência, Barros Barreto, sofre redução de um período para o outro.

No segundo qüinqüênio há uma expansão das áreas "sem domínio", concomitante com o surgimento de área de domínio de unidades menores, indicando os efeitos do processo de municipalização/descentralização bem como da expansão de unidades do PSF. Estes resultados parecem refletir a ação integrada dessas unidades com a de referência em cada DS, ampliando as ações no acompanhamento do tratamento, na detecção pelas orientações e exames de contatos, encaminhando as complicações. Referência especial deve ser feita ao caso de Peixinhos/COHAB (Companhia de Habitação), onde a integração se deu de forma particular, já que três unidades

Casos novos de hanseníase, população estimada e coeficientes de detecção e de prevalência por 10 mil habitantes. Olinda, Pernambuco, Brasil, $1991 / 2000$.

\begin{tabular}{|c|c|c|c|c|c|}
\hline \multirow[b]{2}{*}{ Ano } & \multicolumn{2}{|c|}{ Casos } & \multirow[t]{2}{*}{$\begin{array}{l}\text { População ajustada } \\
\text { (meio do período) }\end{array}$} & \multicolumn{2}{|c|}{$\begin{array}{l}\text { Coeficientes por } 10 \mathrm{mil} \\
\text { habitantes }\end{array}$} \\
\hline & Novos & Registro & & Detecção & Prevalência \\
\hline 1991 & 239 & 296 & 341.127 & 7,0 & 8,7 \\
\hline 1992 & 227 & 338 & 342.735 & 6,6 & 9,9 \\
\hline 1993 & 268 & 424 & 344.350 & 7,8 & 12,3 \\
\hline 1994 & 255 & 490 & 345.974 & 7,4 & 14,2 \\
\hline 1995 & 329 & 664 & 347.604 & 9,5 & 19,1 \\
\hline 1996 & 386 & 826 & 349.243 & 11,1 & 23,7 \\
\hline 1997 & 373 & 968 & 353.783 & 10,5 & 27,4 \\
\hline 1998 & 418 & 1.097 & 358.381 & 11,7 & 30,6 \\
\hline 1999 & 413 & 1.173 & 363.039 & 11,4 & 32,3 \\
\hline 2000 & 427 & 1.386 & 367.758 & 11,6 & 37,7 \\
\hline \multicolumn{6}{|l|}{ Média } \\
\hline no período & 333,5 & - & 348.423 & 9,6 & - \\
\hline
\end{tabular}

Fonte: Sistema de Informações de Agravos de Notificação, Ministério da Saúde/

Centro Nacional de Epidemiologia/Instituto Brasileiro de Geografia e Estatística. 
Estimativa da intensidade de ocorrência da hanseníase no município, atendidos pelas principais unidades de saúde. Olinda, Pernambuco, Brasil, 1991/2000.

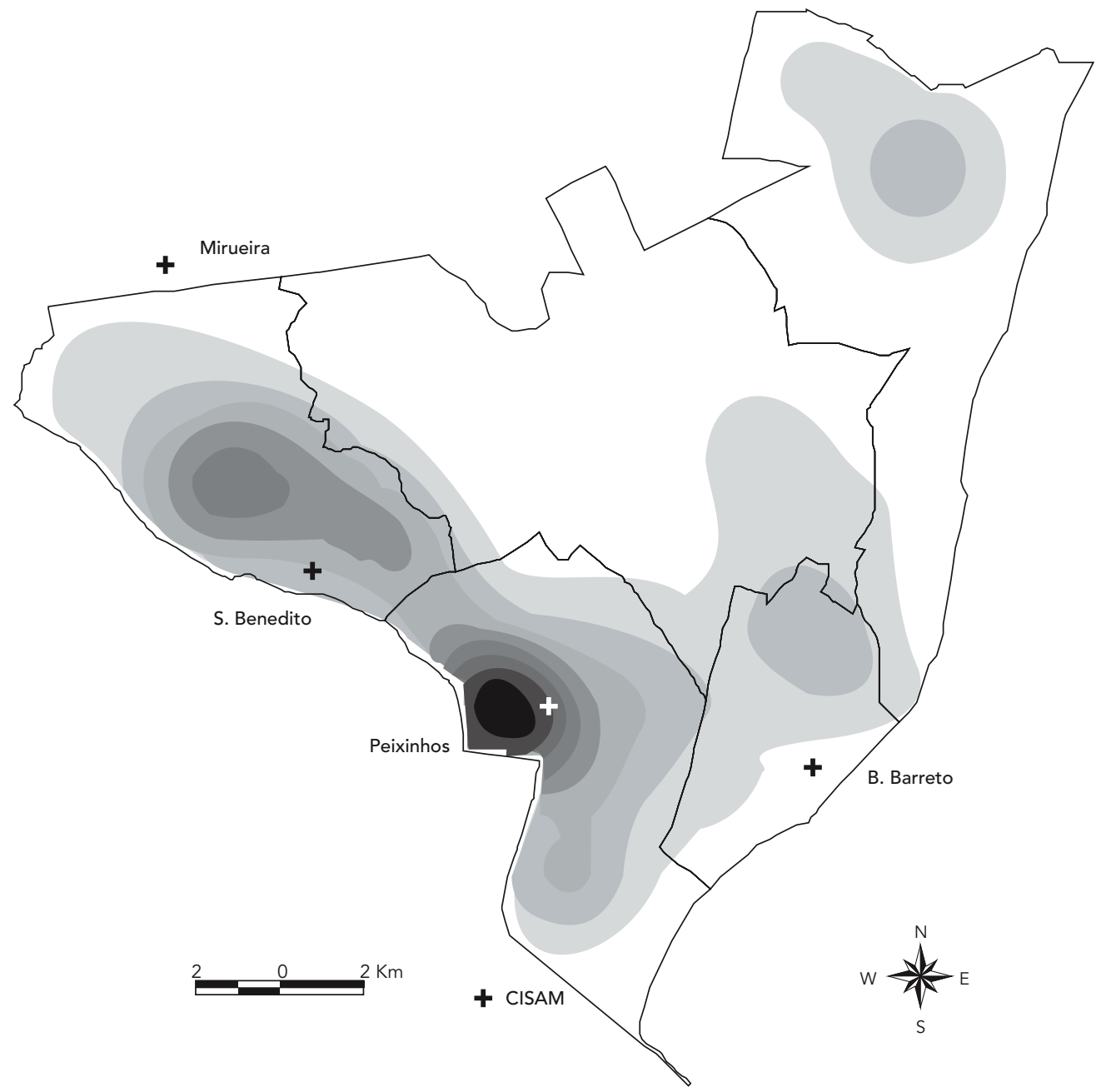

Nota: 2.734 casos georreferenciados; largura de banda $=1.000 ;$ grid = $200 \times 200$, células de $50 \mathrm{~m} \times 50 \mathrm{~m}$.

do PSF usam o mesmo espaço físico da unidade como base, estreitando a integração, explicando a significativa área de domínio surgida. Enfim, identifica-se efeitos de um esforço razoável na busca da organização dos serviços de saúde do município.

Na análise da dinâmica dos atendimentos pelas unidades de saúde onde ocorreram divisões de domínio, com base no segundo par de mapas, que classifica áreas de acordo com as unidades que exercem o maior atendimento, identificouse áreas onde, mesmo não atingindo os $50 \%$, as unidades atenderam quantidades expressivas de casos. Estas unidades podem ser consideradas importantes prestadores de serviços, corroborando com a indicação de efeitos do processo de municipalização.

\section{Discussão}

Uma questão importante para a reorganização dos serviços de saúde, em Olinda, é a necessidade de reverter o atual quadro de "evasão" dos doentes com hanseníase, ali residentes, para serem atendidos em outros municípios. Em torno 
Figura 3

Mapas de áreas de domínio e áreas de máximo atendimento (área em que cada unidade é o maior atendedor). Olinda, Pernambuco, Brasil, 1991/2000.

\section{Áreas de domínio}

a) 1991-1995

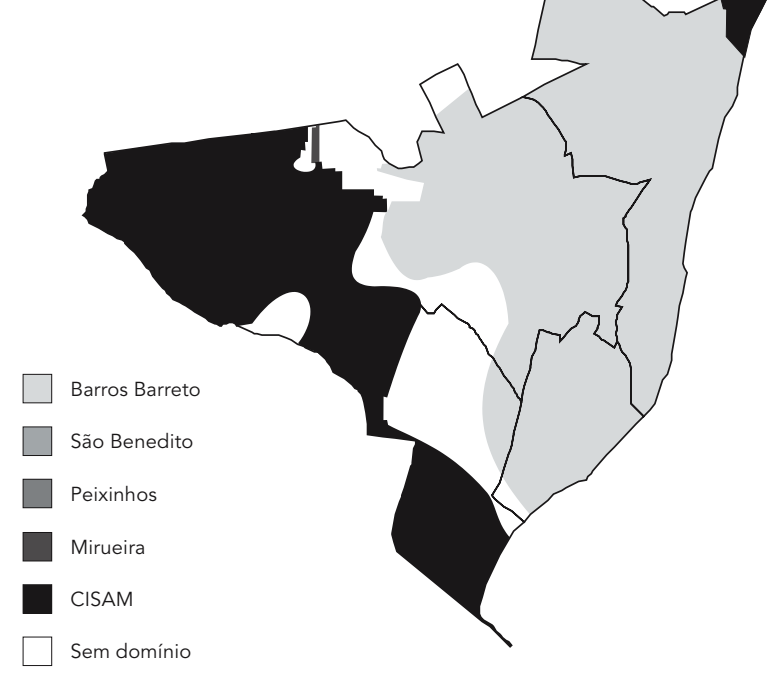

b) $1996-2000$

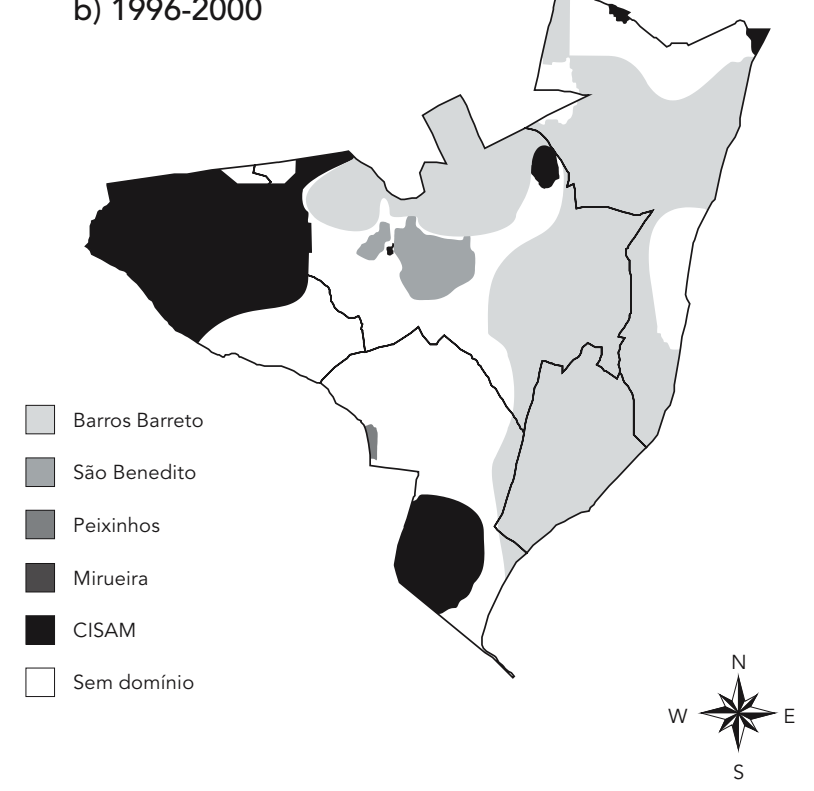

\section{Áreas de máximos atendimentos}

c) $1991-1995$

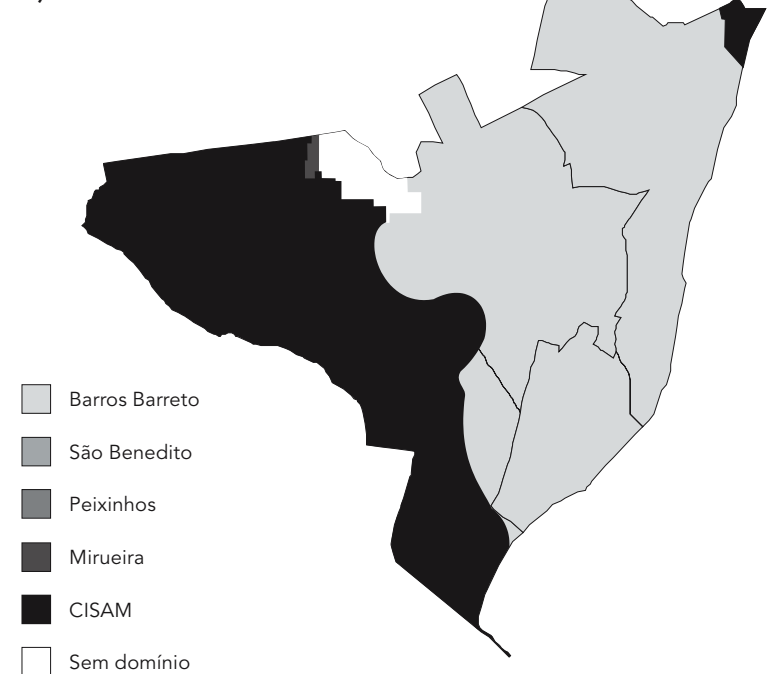

d) $1996-2000$

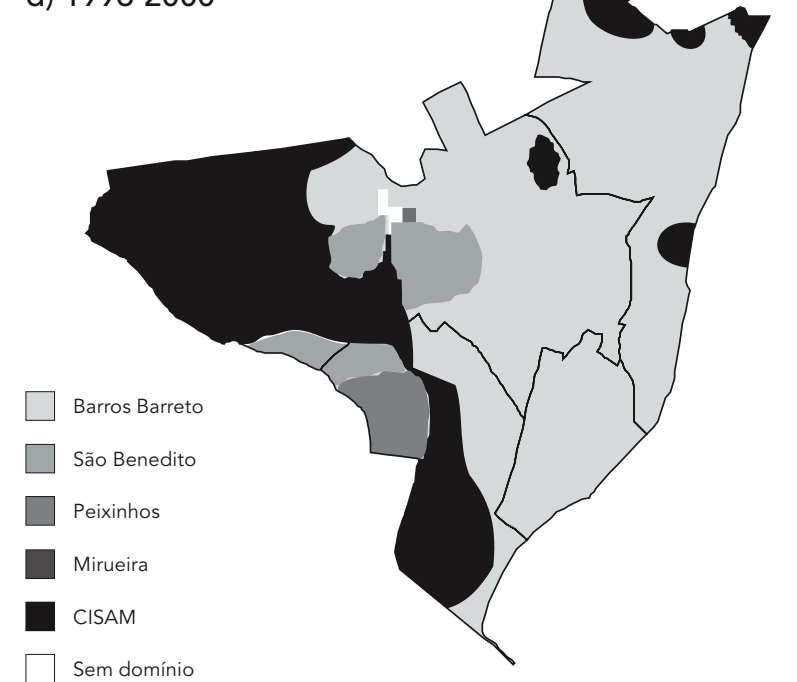

CISAM: Centro Integrado de Saúde Amauri de Medeiros. 
Distribuição da clientela de casos de hanseníase de residentes em Olinda, Pernambuco, Brasil, conforme tipo de demanda, das principais unidades de saúde de atendimento, 1991/2000.

\begin{tabular}{|c|c|c|c|c|c|c|c|c|}
\hline \multirow{4}{*}{$\begin{array}{l}\text { Unidades/ } \\
\text { Municípios }\end{array}$} & & & \multicolumn{6}{|c|}{ Tipo de demanda } \\
\hline & \multirow{2}{*}{\multicolumn{2}{|c|}{$\begin{array}{c}\text { Total } \\
\text { Qüinqüênio }\end{array}$}} & \multirow{2}{*}{\multicolumn{2}{|c|}{$\begin{array}{l}\text { Espontânea } \\
\text { Qüinqüênio }\end{array}$}} & \multirow{2}{*}{\multicolumn{2}{|c|}{$\begin{array}{c}\text { Encaminhamento } \\
\text { Qüinqüênio }\end{array}$}} & \multirow{2}{*}{\multicolumn{2}{|c|}{$\begin{array}{c}\text { Outro } \\
\text { Qüinqüênio }\end{array}$}} \\
\hline & & & & & & & & \\
\hline & 1으 & 2oㅇ & 1으 & 2o & 1으 & 2o & 1으 & 2으 \\
\hline Barros Barreto & 424 & 577 & 207 & 97 & 40 & 384 & 177 & 96 \\
\hline São Benedito & 167 & 352 & 70 & 170 & 9 & 134 & 88 & 48 \\
\hline Peixinhos & 0 & 182 & 0 & 44 & 0 & 30 & 0 & 108 \\
\hline Olinda & 591 & 1.111 & 277 & 311 & 49 & 548 & 265 & 252 \\
\hline Mirueira & 50 & 61 & 40 & 18 & 7 & 39 & 3 & 4 \\
\hline \multicolumn{9}{|l|}{ Centro Integrado de } \\
\hline Saúde Amauri de Medeiros & 581 & 713 & 439 & 76 & 88 & 488 & 54 & 149 \\
\hline Outros municípios & 631 & 774 & 479 & 94 & 95 & 527 & 57 & 153 \\
\hline Total & 1.222 & 1.885 & 756 & 405 & 144 & 1.075 & 322 & 405 \\
\hline
\end{tabular}

de $45 \%$ desses casos foram atendidos no Recife e desses, $92 \%$ (1.294) foram atendidos no CISAM (Centro Integrado de Saúde Amauri de Medeiros), considerando-se todo o período do estudo. Como mostra os números na Tabela 2, Olinda apresenta significativo crescimento nos atendimentos, saltando de 591 no primeiro qüinqüênio para 1.111 (58,9\%) no segundo, refletindo a mudança do perfil de atendimentos de um período para outro. Quanto à demanda, os números também registram uma mudança, mostrando que o crescimento da demanda por encaminhamento é bem mais expressiva, saltando de 49 para 548, quase $50 \%$ dos casos atendidos por Olinda.

A construção de mapas identificando áreas a partir da utilização efetiva dos serviços de saúde pela população permitiu visualizar os "efeitos" da municipalização, notadamente a reorganização do padrão espacial das áreas de domínio de atendimento, com a redução da evasão de casos para outros municípios. Conseqüentemente observou-se um significativo aumento do atendimento por importantes unidades municipais, apresentando perfil de maior concentração nos seus entornos, em áreas de grande intensidade de ocorrência da doença, como nas áreas de Peixinhos e São Benedito, que parecem refletir a territorialização dos atendimentos dos casos de hanseníase. A construção de mapas possibilitou ainda a identificação das mudanças necessárias do sistema de saúde ainda não efetuadas.
Nos mapas dos "domínios" de atendimentos das unidades, áreas onde uma unidade atende mais de 50\% dos casos ali residentes (Figura 3), identifica-se que ocorre uma divisão de áreas entre as maiores unidades prestadoras. Todo o Distrito 2 é praticamente domínio de sua unidade de referência Barros Barreto enquanto que boa parte do Distrito 1 é domínio do CISAM, e praticamente o restante corresponde a uma área sem domínio de algum serviço de saúde.

No caso do Distrito 2, o resultado parece atender ao enfoque da territorialização da clientela, considerando a proposta de tomar a necessidade da população como o "norte" que deve orientar a oferta organizada dos serviços, rompendo com o modelo assistencial calcado na demanda espontânea.

Idealmente as unidades destinadas e estruturadas para atender a demanda de uma determinada área deveriam exercer o domínio de atendimentos aos casos da doença em seus residentes. Como resultado, ocorreria um predomínio da unidade no atendimento de casos de hanseníase em residentes na área de domínio, cuja intensidade poderá ser dimensionada, avaliando o grau com que exerce este domínio e conseqüentemente a possível competição com outros serviços, procurando medir a participação de outras unidades que dividem os atendimentos na área.

Assim, possíveis divisões de atendimento poderão ser consideradas no planejamento das 
ações de saúde, com melhor detalhamento da origem da clientela e da oferta de serviços. Do ponto de vista dos gestores dos distritos sanitários deve-se levar em consideração a situação de saúde como um todo e não apenas um só agravo ou endemia.

Observa-se ainda, analisando os mapas de domínio de atendimento da Figura 3, que no segundo qüinqüênio há uma redução das áreas de domínio de Barros Barreto e do CISAM, ocorrendo sensível ampliação das áreas sem domínio, evidenciando uma mudança de comportamento dos atendimentos. Essas imagens expressam, como já descrito anteriormente, o aumento da participação nos atendimentos por outras unidades, quais sejam São Benedito e COHAB/Peixinhos, porém, ainda não suficientes para atingir o "domínio" da área.

O segundo par de mapas da Figura 3, que complementam a análise apresentada que se referiu ao primeiro par, e que é feita a partir do critério estabelecido anteriormente, ou seja, exerce o domínio de atendimento em determinada área aquela unidade que efetuou atendimentos na ordem de $50 \%$ ou mais dos casos da área, permite detalhar a análise da evolução do atendimento, considerando especialmente áreas onde ocorreu divisão de clientela. As imagens permitiram identificar áreas onde mesmo não atingindo os 50\%, as unidades atendem expressiva quantidade de casos, podendo ser consideradas importantes prestadoras de atendimentos da área.

A partir de 1995, ano do início do processo de municipalização do Programa de Controle da Hanseníase, observa-se que boa parte da área identificada como sem domínio tem como maior prestador São Benedito. Destaca-se também a unidade de Peixinhos, apresentando-se com maior prestação de atendimentos aos residentes no seu entorno, atestando a evolução de desempenhos, reduzindo a influência do CISAM.

É oportuno considerar que a implementação de equipes do PSF pode ter contribuído na reorganização da assistência aos pacientes com hanseníase e para uma melhor organização da demanda, ampliando a capacidade da população em perceber a doença, saber que pode ser curado, ir a um serviço de saúde, ser diagnosticado e tratado. A atuação do PSF pode ter contribuído para melhorar o acesso da população aos serviços de saúde, de um modo geral, lembrando que no segundo qüinqüênio cresceu consideravelmente a demanda por "encaminhamentos" para as unidades de saúde do município.

A construção de áreas de domínio de atendimento das unidades na lógica do acesso, ou seja, considerando a origem dos casos e a unidade onde ocorreu a assistência, delimitou áreas ou estratos populacionais circunscritos à unidade de saúde que exerceu o predomínio de atendimento. Essa abordagem permitiu a compreensão da dinâmica da oferta e da procura dos serviços e pode vir a dar suporte à organização do setor, visando à implementação de sistemas de vigilância da saúde.

A construção dessas áreas de domínio de atendimentos das unidades, estabelecendo o corte qüinqüenal serviu como um importante instrumento para visualizar o impacto do processo de municipalização no que se refere às atividades de controle da hanseníase no Município de Olinda.

O uso da técnica da distribuição espacial aplicada ao caso da hanseníase na área do estudo permitiu identificar que o problema não se dá de forma homogênea. Do mesmo modo, foi possível observar diferenças entre subáreas, relativas aos focos da doença, que podem orientar estratégias e ações para o seu controle.

A qualificação de áreas com base nas intensidades da ocorrência da doença, definindo estratos que representam territórios geográficos onde grupos populacionais procuraram prioritariamente uma determinada unidade de saúde, permite qualificá-las e enxergar o município em partes onde as ações podem ser planejadas em harmonia com critérios de necessidades e prioridades, e dar ao nível local suporte de fundamental importância, guardando coerência com as diretrizes do Sistema Único de Saúde. 


\section{Resumo}

O desafio para redução da prevalência da Hanseníase endemia de grande importância no cenário brasileiro, tem se fundamentado na descentralização para os serviços de atenção básica. Descreveu-se inicialmente a estruturação e a oferta dos serviços para o atendimento de casos de hanseníase. Em seguida analisou-se a evolução dos indicadores epidemiológicos e operacionais, tomando como referência os períodos pré e pós a municipalização. Por fim, a análise espacial permitiu identificar a distribuição territorial da ocorrência da endemia e analisar o padrão de áreas geográficas construídas segundo o atendimento pelas unidades de saúde e sua evolução. A partir da localização geográfica dos centróides dos setores censitários de residência, e usando técnica de alisamento espacial, com base na estimativa de Kernel, foram construídas áreas de domínio de atendimentos de cada unidade. Após a municipalização, observa-se aumento da detecção e tratamento pelo município, reduzindo a evasão a outros municípios, mudanças no comportamento da demanda, com aumento da clientela referida no uso dos serviços, e alterações importantes nos indicadores epidemiológicos e operacionais.

Hanseníase; Análise Espacial; Acesso aos Serviços de Saúde

\section{Colaboradores}

T. M. Lapa contribuiu na construção do objeto de estudo, definição dos objetivos e metodologia, levantamento de dados, processamento, análise e redação do artigo. M. F. P. M. Albuquerque e M. S. Carvalho participaram na análise e interpretação dos resultados e revisão do texto. J. C. Silveira Júnior colaborou no processamento e análise dos dados, especialmente quanto ao uso das técnicas de geoprocessamento e análise espacial.

\section{Referências}

1. Ministério da Saúde. Guia para o controle da hanseníase. Brasília: Departamento de Atenção Básica, Secretaria de Políticas de Saúde, Ministério da Saúde; 2002.

2. Organização Pan-Americana da Saúde. Hanseníase. http://www.opas.org.br/programas/hanseníase/hansen.htm (acessado em 05/Fev/2004).

3. Rezende FAVS, Almeida RMV, Nobre FF. Diagramas de Voronoi para a definição de áreas de abrangência de hospitais públicos no Município do Rio de Janeiro. Cad Saúde Pública 2000; 16:467-75.

4. Souza WV, Barcellos CC, Brito AM, Carvalho MS, Cruz OG, Albuquerque MFM, et al. Aplicação de modelo bayesiano empírico na análise espacial da ocorrência de hanseníase. Rev Saúde Pública 2001; 35:474-80.

5. Lapa TM, Ximenes R, Silva N, Souza W, Albuquerque MFM, Campozana G. Vigilância da hanseníase em Olinda, Brasil, utilizando técnicas de análise espacial. Cad Saúde Pública 2001; 17:1153-62.

6. Mencaroni DA, Pinto Neto JM, Villa TCS, Oliveira MHP. Análise espacial da endemia hansênica na área urbana do Município de Ferdanópolis/SP. Hansen Int 2004; 29:12-20.

7. Montenegro ACD, Werneck GL, Kerr-Pontes LRS, Barreto ML, Feldmeier H. Spatial analysis of the distribution of leprosy in the state of Ceará, Northeast Brazil. Men Inst Oswaldo Cruz 2004; 99:683-6.

8. Starfield B. Atenção primária: equilíbrio entre necessidades de saúde, serviços e tecnologia. Brasília: Organização das Nações Unidas para a Educação, a Ciência e a Cultura/Ministério da Saúde; 2002.

9. Oliveira EXG, Travassos C, Carvalho MS. Acesso à internação hospitalar nos municípios brasileiros em 2000: territórios do Sistema Único de Saúde. Cad Saúde Pública 2004; 20:S298-309.

10. Kadt E, Tasca R. Promovendo a eqüidade: um novo enfoque com base no setor da saúde. São Paulo: Editora Hucitec; 1993.

11. Pinheiro RS. Estudos sobre variações no uso de serviços de saúde: abordagens metodológicas e a utilização de grandes bases de dados nacionais [Tese de Doutorado]. Rio de Janeiro: Escola Nacional de Saúde Pública, Fundação Oswaldo Cruz; 1999.

12. Lovett A, Haynes R, Sünnenberg G,Gale S. Car travel time and accessibility by bus to general practitioner services: a study using patient registers and GIS. Soc Sci Med 2002; 55:97-111.

13. Oliveira EXG, Carvalho MS, Travassos C. Territórios do Sistema Único de Saúde: mapeamento das redes de atenção hospitalar. Cad Saúde Pública 2004; 20:386-402.

14. Pinheiro RS, Travassos C, Gamerman D, Carvalho MS. Mercados hospitalares em área urbana: uma abordagem metodológica. Cad Saúde Pública 2001; 17:1111-21.

15. Bailey TC .The analysis of point patterns. In: Bailey TC, Gatrell AC, editors. Interative spatial data analysis. Essex: Longman Scientific \& Technical; 1995. p. 75-139.

Recebido em 05/Set/2005

Versão final reapresentada em 08/Dez/2005 Aprovado em 14/Dez/2005 